\title{
"RAS"ling $\beta$ cells to proliferate for diabetes: why do we need MEN?
}

\author{
Adolfo García-Ocaña and Andrew F. Stewart \\ The Diabetes, Obesity and Metabolism Institute and The Division of Endocrinology and Bone Disease, Icahn School of Medicine, Mount Sinai, New York, USA.
}

\begin{abstract}
Adult human pancreatic $\beta$ cells are refractory to current therapeutic approaches to enhance proliferation. This reluctance to expand is problematic, especially for people with diabetes who lack sufficient numbers of functional insulin-producing $\beta$ cells and could therefore benefit from therapies for $\beta$ cell expansion. In this issue of the $J C l$, Chamberlain et al. describe a surprising series of observations that involve two downstream arms of the RAS signaling pathway, MAPK and RASSF proteins, which also involve the tumor suppressor menin. The findings of this study may help explain the difficulty of inducing $\beta$ cell proliferation and may provide leads for therapeutic expansion of human $\beta$ cells.
\end{abstract}

\section{Diabetes and the loss of $\beta$ cell function}

Diabetes affects 330 million people worldwide, and twice as many individuals have metabolic syndrome. Type 1 diabetes (T1D) is due to autoimmune loss of insulin-producing pancreatic $\beta$ cells, while type 2 diabetes (T2D) results from a combination of lifestyle choices and genetics. Historically, T2D has been viewed as a disease of Western countries; however, T2D is now highly prevalent in counties such as China and India, which have surpassed the US in the number of people with diabetes. While it has long been appreciated that T2D is associated with insulin resistance, only recently have GWAS and autopsy studies indicated that T2D is also due in large part to inadequate numbers of functional pancreatic $\beta$ cells. Thus, pancreatic $\beta$ cell dysfunction and/or deficiency underlie both major forms of diabetes mellitus (DM). In terms of public health, this attention to the $\beta$ cell is important, because some $30 \%$ of hospital beds in the US are occupied by people with diabetes and its complications, which include heart attack, stroke, renal failure, blindness, neuropathy, peripheral vascular disease with limb amputation, and now liver transplantation. It is estimated by the American Diabetes Association that $\$ 245$ billion is spent annually in the US on diabetes, its complications, and lost productivity. We have a large problem on our collective hands.

A solution would either be to generate more $\beta$ cells in situ or to replace them from ex vivo sources, such as through expansion of $\beta$ cells from cadaveric pancreatic islet donors, from human stem cells, or from xenograft sources such as porcine islets. Fifteen to twenty years ago, the possibility of generating replacement $\beta$ cells from any of the above sources was viewed as largely impossible, but over the past decade, progress has been made in many of these areas. In mouse and rat models, multiple approaches, including administration of growth factors, nutrients, signaling molecules, high-fat diet, and transgene expression, have been shown to rapidly and robustly increase $\beta$ cell proliferation and mass. Unfortunately, we are now learning that this is more difficult in adult human $\beta$ cells. Thus, at present, we have a desperate - but so far unmet need to understand why adult human $\beta$ cells are so refractory to replication

Related Article: p. 4093

Conflict of interest: The authors have declared that no conflict of interest exists.

Reference information: J Clin Invest. 2014;124(9):3698-3700. doi:10.1172/JCI77764.

and to develop small-molecule or peptide approaches to expand $\beta$ cell mass in people with diabetes or expand these cells ex vivo for replacement therapies.

\section{$\beta$ cell proliferation: of K-RAS and MEN}

In this issue of the JCI, Chamberlain et al. (1) describe a string of unexpected results that provide insight into why human $\beta$ cells are so refractory to proliferation and provide leads toward therapeutic human $\beta$ cell expansion. Chamberlain and colleagues were interested in the small GTPase K-RAS, an oncogenic protein that is causally associated with many cancers, including carcinomas of the pancreas, lung, and colon, to name a few. Based on the clear oncogenic role of K-RAS in so many tissue types, it would be predicted that loss of K-RAS reduces proliferation in affected cells. Surprisingly, Chamberlain et al. found that $\beta$ cells in mice lacking one Kras allele developed hyperplasia, with a marked increase in $\beta$ cell neogenesis. Conversely, expression of a constitutively active form of K-RAS reduced both $\beta$ cell proliferation and overall numbers. Thus, in contrast to pancreatic adenocarcinoma, in which K-RAS drives proliferation, active $\mathrm{K}-\mathrm{RAS}$ is a cell-cycle inhibitor in pancreatic $\beta$ cells.

To better understand how K-RAS inhibits pancreatic $\beta$ cell growth, Chamberlain and colleagues (1) explored the balance between two downstream signaling arms of the RAS family: RAF/MAPK signaling and the RASSF tumor-suppressor family (Figure 1). Neuroendocrine tumors have been described as having loss or inactivation of RASSF1A; therefore, Chamberlain et al. queried whether RASSF1A might be phosphorylated and activated as a growth inhibitor in the presence of K-RAS activation. Indeed, activation of K-RAS in $\beta$ cells led to activation of RAS/MAPK mitogenic signaling as well as RASSF1A antimitogenic signaling. It is very surprising that the inhibitory effects of active RASSF1A in 
A

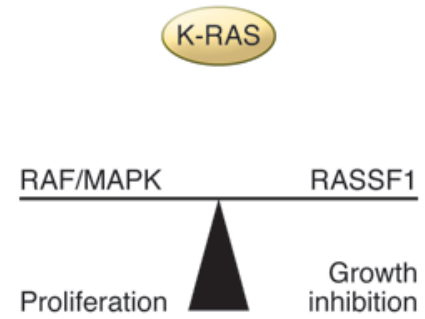

Net $=$ replicative quiescence
B

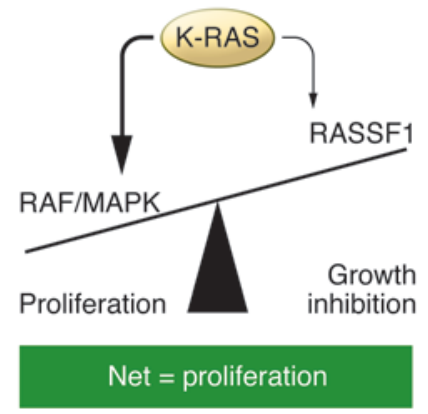

e.g., pancreatic cancer
C



Net $=$ growth inhibition

e.g., pancreatic $\beta$ cell

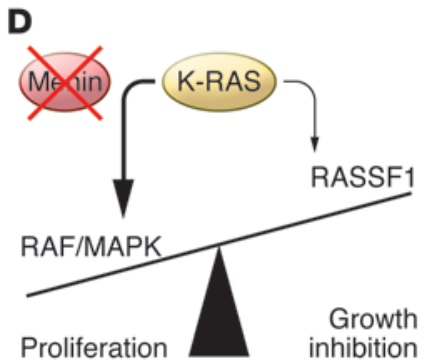

Net $=$ proliferation

e.g., MEN1 endocrine cell

Figure 1. The balancing act downstream of K-RAS signaling determines cell proliferation. (A) Representation of typical cells in which K-RAS and its two downstream arms are not activated. (B) In most tissues, including pancreatic cancer cells, activation of K-RAS results in activation of downstream targets, including the RAF/MAPK pathway, which generally drives proliferation, and the tumor-suppressor family of RASSF proteins. K-RAS activates both pathways, but the mitogenic balance swings in favor of proliferation, as occurs in pancreatic adenocarcinoma. (C) In contrast, in pancreatic endocrine $\beta$ cells, K-RAS activation drives both pathways, but the mitogenic RAF/MAPK arm is inhibited by the downstream tumor suppressor menin; therefore, the proliferation-inhibiting RASSF arm tips the balance in favor of inhibition of proliferation. (D) In menin-deficient $\beta$ cells, the balance swings back in favor of proliferation. For a more detailed biochemical schematic, see Figure 5J in Chamberlain et al. (1). This paradigm appears to apply to other endocrine cells such as pituitary and parathyroid cells.

$\beta$ cells can override the mitogenic effects of activated RAS/MAPK signaling. But why is this the case specifically in $\beta$ cells?

Given that rampant proliferation occurs in three endocrine tissues - islet, pituitary, and parathyroid cells - in people with the multiple endocrine neoplasia type 1 (MEN1) syndrome as the result of inactivating mutations in both alleles of the MEN1 gene, Chamberlain and colleagues wondered whether the MEN1 gene product menin might also play a cell-specific role in the K-RAS regulation of $\beta$ cell proliferation. Examination of parathyroid and pituitary tissues from mice heterozygous for Kras revealed hyperplasia in both endocrine tissues, similar to that observed in humans with MEN1. Buoyed by this result, Chamberlain and colleagues explored the combined effect of heterozygous Men deletion with constitutive activation of K-RAS on murine $\beta$ cell proliferation (1). Men haploinsufficiency relieved the inhibitory effects of K-RAS activation; therefore, K-RAS now actually enhanced, rather than inhibited, $\beta$ cell proliferation. Remarkably, menin appears to be the $\beta$ cell-specific inhibitor of K-RAS signaling. Menin expression in $\beta$ cells effectively alters the outcome of K-RAS signaling from promitogenic, mediated by activated RAF-MAPK signaling, to antiproliferative, mediated by activated RASSF1A.

Excitingly, menin-dependent differences in the outcome of K-RAS signaling provide a possibility for translation into pharmacologic therapy. Based on the role of K-RAS in cancer cell proliferation, most would probably have guessed that RAS inhibitors would interfere with, or have no effect on, $\beta$ cell proliferation. In contrast to intuition, Chamberlain et al. determined that inhibition of RAS with a combination of farnesyl transferase inhibitor (FTI) and geranylgeranyl transferase inhibitor (GGTI) mimic the effects of allelic Kras loss (1). Importantly, the FTI-GGTI combination also induced proliferation in human $\beta$ cells, increasing $\beta$ cell proliferation from $\sim 0.1 \%$ to $\sim 0.5 \%$ in isolated human pancreatic islets.

The connection between RASSF1 and $\beta$ cell proliferation was another unanticipated result. While it is true that RASSF1A can function as a tumor suppressor and has been linked to neuroendocrine tumors, a link to $\beta$ cell proliferation has not been on the collective minds of the $\beta$ cell research community. Apparently, it should be.

Yet another surprise result provided by Chamberlain et al. is the demonstration that menin inhibits RAS/RAF/MAPK signaling (1). While the loss of menin was shown to be central to the MEN1 syndrome some 25 years ago $(2,3)$, there is not a clear picture of what precisely menin does. Menin dysfunction is clearly associated with endocrine tumors, but the vast majority of these are not $\beta$ cell tumors, or even islet tumors. It also is unknown why people who harbor germline MEN1 mutations do not develop tumors in all tissues, since MEN1 is expressed ubiquitously. Menin is a nuclear protein that transcriptionally activates cell-cycle inhibitors, such as p16INK ${ }^{4 a}$, $\mathrm{p} 18 \mathrm{INK}^{4 \mathrm{c}}$, and $\mathrm{p} 27^{\mathrm{KIP}}$, and a member of the trithorax histone methylation complex that also includes methyltransferases MLL1 and MLL2 (3-7). Presumably, these cell-cycle inhibitors have effects on many, if not all, cell types; therefore, it remains uncertain as to why menin loss specifically affects endocrine cells. Thus, menin, like RASSF1, appears to be a key inhibitor of RAS signaling in $\beta$ cells. Precisely how and whether menin can be manipulated in a $\beta$ cell-specific manner is unknown, though the observation by Chamberlain et al. that the small-molecule menin-MLL inhibitor MI- 2 enhanced human $\beta$ cell proliferation provides hope in this regard.

While it may be a surprise to those in other fields, the modest proliferation achieved by Chamberlain and colleagues will not be surprising to the $\beta$ cell cognoscenti. The good news is that proliferation was induced in up to $1 \%$ of human $\beta$ cells, but the painful reality is that the remaining $99 \%$ of $\beta$ cells do not want to join in the replicative symphony. Thus, we are left with the hope of finding novel molecules to induce human $\beta$ cells to replicate and the frustration that only a very select few $\beta$ cells choose to participate.

\section{Conclusions and future directions}

The report by Chamberlain et al. is timely (1). Current $\beta$ cell research is clearly focused on developing ways to drive 
human $\beta$ cell regeneration, as emphasized by funding agencies, including the National Institute of Diabetes and Digestive and Kidney Diseases (NIDDK) and the Juvenile Diabetes Research Foundation (JDRF), as well as pharmaceutical and biotechnology companies. The K-RAS/ menin/RASSF1A story comes amidst an increasing number of tantalizing, but sofar unpublished, reports at diabetes conferences that disclose novel molecules and pathways that induce human $\beta$ cell replication. The rates of replication are low, with labeling indices of $0.5 \%$ to $1.0 \%$, but the reports have uncovered a remarkable number of different pathways that appear to be important for $\beta$ cell replication, suggesting that a combinatorial approach of $\beta$ cell regenerative strategies may be useful.

There are a number of current challenges to translating $\beta$ cell replicative therapies to clinical use. First, we do not understand why the majority of human $\beta$ cells cannot be coaxed to enter into the cell cycle. Is this resistance genetic, epigenetic, inextricably linked to differentiation, miRNA regulated, or mediated by long, noncoding RNAs? Second, we do not know what a "therapeutic rate" or required duration of $\beta$ cell replication might be. For example, would increasing the proliferation rate to $1 \%$ to $3 \%$, a level all humans experience as neonates, for one year be enough to restore $\beta$ cell function in a person with diabetes (8-10)? Third, there are no effective methods to target mitogenic molecules to $\beta$ cells in humans. The FTI-GGTI inhibitors and MI-2 used by Chamberlain et al. can be expected to affect many tissues, because their targets, farnesyl transferase, geranylgeranyl transferase, and menin/MLL are widely expressed. In fact, we currently lack methods to target any molecule specifically to $\beta$ cells; therefore, the identification of cellsurface targets that are unique to human $\beta$ cells will be imperative to permit $\beta$ cell-specific drug delivery. Fourth, a major concern for the development of human $\beta$ cell-specific therapies is the paucity of human $\beta$ cells available to researchers. Currently, the rate of progress in human $\beta$ cell replication research is constrained by the insufficient supply of human cadaveric islets.

In the larger picture of advancing toward the goal of $\beta$ cell replication therapies, the Chamberlain report moves the ball a few more important yards down the field. We now have what will surely be one of several distinct small-molecule or biologic approaches to enhance induction of human $\beta$ cell proliferation. Additional advances are waiting in the wings.

\section{Acknowledgments}

This work was supported by NIDDK grants R-01 DK55023 and U-01 DK089538 (to A.F. Stewart) and R-01s DK067351 and DK077096 (to A. García-Ocaña); JDRF grants 17-2011-598 and 1-2011-603 (to A.F. Stewart); and ADA grant 1-14-BS-069 (to A. García-Ocaña).

Address correspondence to: Adolfo GarcíaOcaña or Andrew F. Stewart, Diabetes, Obesity and Metabolism Institute, Icahn School of Medicine at Mount Sinai, Atran 5,
Box 1152, 1 Gustave L. Levy Place, New York, New York 10029, USA. Phone: 212.241.7680;E-mail:adolfo.garcia-ocana@ mssm.edu (A. García-Ocaña), andrew. stewart@mssm.edu (A.F. Stewart).

1. Chamberlain CE, et al. Menin determines K-RAS proliferative outputs in endocrine cells. J Clin Invest. 2014;124(9):4093-4101.

2. Thakker R, Multiple Endocrine neoplasia type 1. In: DeGroot L, Jameson L, eds. Endocrinology. 6th ed. Philadelphia, Pennsylvania, USA: Saunders Elsevier; 2010:2719-2741.

3. Chandrasekharappa SC, et al. Positional cloning of the gene for multiple endocrine neoplasia type 1. Science. 1997; 276(5311):404-407.

4. Crabtree JS, et al. Of mice and MEN1: insulinomas in a conditional mouse knockout. Mol Cell Biol. 2003;23(17):6075-6085.

5. Matkar S, Thiel A, Hua X. Menin: a scaffold protein that control gene expression and cell signaling. Trends Biochem Sci. 2013;38(8):394-402.

6. Karnick SK, et al. Menin regulates pancreatic islet growth by promoting histone methylation and expression of genes encoding p27kip1 and p18Ink4cs. Proc Nat Acad Sci U S A. 2005;102(41):14659-14664.

7. Karnik SK, et al. Menin controls growth of pancreatic $\beta$ cells in pregnant mice and promotes gestational diabetes mellitus. Science. 2007;318(5851):806-809.

8. Gregg BE, et al. Formation of a human $\beta$ cell population within pancreatic islets is set early in life. J Clin Endocrinol Metab. 2012;97(9):3197-3206.

9. Meier JJ, et al. $\beta$ Cell replication is the primary mechanism subserving the postnatal expansion of $\beta$ cell mass in humans. Diabetes. 2008;57(6):1584-1594.

10. Kassem SA, Ariel I, Thornton PS, Scheimberg I, Glaser B. $\beta$ Cell proliferation and apoptosis in the developing normal human pancreas and in hyperinsulinism of infancy. Diabetes. 2000;49(8):1325-1333. 